CVIA

REVIEW ARTICLE

pISSN 2508-707X / elSSN 2508-7088 https://doi.org/10.22468/cvia.2018.00052 CVIA 2018;2(2):76-84

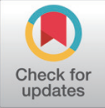

\title{
Cardiac Strain Analysis Using Cine Magnetic Resonance Imaging and Computed Tomography
}

\author{
Michinobu Nagao', Yuzo Yamasaki \\ 'Department of Diagnostic Imaging \& Nuclear Medicine, Tokyo Women's Medical University, \\ Tokyo, Japan \\ 2Department of Molecular Imaging \& Diagnosis, Graduate School of Medical Sciences, \\ Kyushu University, Fukuoka, Japan
}

\begin{abstract}
Using tagged cine magnetic resonance imaging (MRI) to analyze myocardial strain provides direct information regarding the timing of onset and peak of myocardial contraction and allows for the evaluation of regional function and mechanical dyssynchrony. Cardiac MRI lacks the disadvantages of acoustic windows and a narrow field of view, with the advantage of measuring right ventricular strain and the geometric difference in left ventricular contraction. Previous studies using tagged MRI demonstrated that a contraction delay between the right and left ventricles correlated with right ventricular dysfunction in adults with congenital heart disease and pulmonary hypertension, and that spatial dyssynchrony was associated with a reduction in cardiac sympathetic activity in non-ischemic heart failure. Recently, cardiac computed tomography (CT) was also found to enable the quantitative assessment of two-dimensional and three-dimensional myocardial strain. This review focuses on strain analysis using tagged MRI and its current post-processing methods, known as feature tracking, discusses upcoming CT-based methods of strain analysis, and introduces their clinical applications.
\end{abstract}

Key words Myocardial strain · Cardiac magnetic resonance imaging · Tagged imaging Feature tracking $\cdot$ Computed tomography.

Received: March 7, 2018

Revised: March 24, 2018

Corresponding author

Michinobu Nagao, MD, PhD

Department of Diagnostic Imaging and

Nuclear Medicine,

Tokyo Women's Medical University,

8-1 Kawada-cho, Shinjuku-ku,

Tokyo 162-8666, Japan

Tel: 81-3-3353-8111

Fax: 81-3-5269-9247

E-mail: nagao.michinobu@twmu.ac.jp

\section{INTRODUCTION}

Myocardial contractility is an essential determinant of ventricular function. The left ventricular (LV) ejection fraction (EF) has been used as a global index of ventricular systolic function. However, LVEF is influenced by ventricular geometry and loading conditions, which may remain unchanged in affected patients until the underlying disease process has advanced significantly. Myocardial strain, a sensitive measure of deformation, is defined as the relative change in fiber length from end diastole. While measuring myocardial strain in vivo would require a precise knowledge of the direction in which local fibers lie, clinical imaging modalities circumnavigate this challenge by measuring strain in three principal directions (radial, circumferential, and longitudinal) relative to the central axis of the ventricle. Regional myocardial strain may be an earlier marker of incipient myocardial dysfunction [1]. Cardiac-tagged magnetic resonance

(c) This is an Open Access article distributed under the terms of the Creative Commons Attribution Non-Commercial License (http://creativecommons.org/licenses/by$\mathrm{nc} / 4.0$ ) which permits unrestricted non-commercial use, distribution, and reproduction in any medium, provided the original work is properly cited. imaging (MRI) is considered to be the reference method for measuring myocardial strain [2]. However, the clinical focus for noninvasive deformation imaging is currently moving from tailored acquisitions, such as myocardial tagging, to the postprocessing of standard cine imaging and multi-phase computed tomography (CT) imaging, resulting in increased accessibility and availability. Speckle tracking echocardiography has become the strain imaging of choice because additional imaging is not required and post-processing is easier. Feature-tracking cardiac MRI (FT-CMR), which mimics speckle tracking echocardiography, can potentially be used more widely than tagging MRI. This review focuses on strain analysis using classical tagged MRI and the current status of post-processing methods for these images, known as feature tracking, in addition to discussing upcoming CT-based methods of strain analysis and introducing their clinical applications. Table 1 summarizes strengths and weaknesses of these methods of strain analysis. 
Table 1. Strain analysis characteristics

\begin{tabular}{|c|c|c|c|c|c|c|c|}
\hline & Principle & Dimension & Object analysis & $\begin{array}{c}\text { Spatial } \\
\text { resolution }\end{array}$ & $\begin{array}{l}\text { Temporal } \\
\text { resolution }\end{array}$ & $\begin{array}{c}\text { Post- } \\
\text { processing }\end{array}$ & $\begin{array}{c}\text { Cardiac } \\
\text { device }\end{array}$ \\
\hline Tagged-CMR & HARP & $2 \mathrm{D}$ & Segmental deformation & $5-8 \mathrm{~mm}$ & 20-50 frame/cycle & + & Non-adaptive \\
\hline FT-CMR & Tissue tracking & $2 \mathrm{D}$ & Myocardial contour & $8-10 \mathrm{~mm}$ & 20-30 frame/cycle & - & Non-adaptive \\
\hline CT strain & Motion coherence & $3 \mathrm{D}$ & Voxel movement & High & 10-20 frame/cycle & + & Adaptive \\
\hline
\end{tabular}

CMR: cardiac magnetic resonance imaging, FT-CMR: feature-tracking cardiac MRI, CT: computed tomography, HARP: harmonic phase,

2D: two-dimensional, 3D: three-dimensional

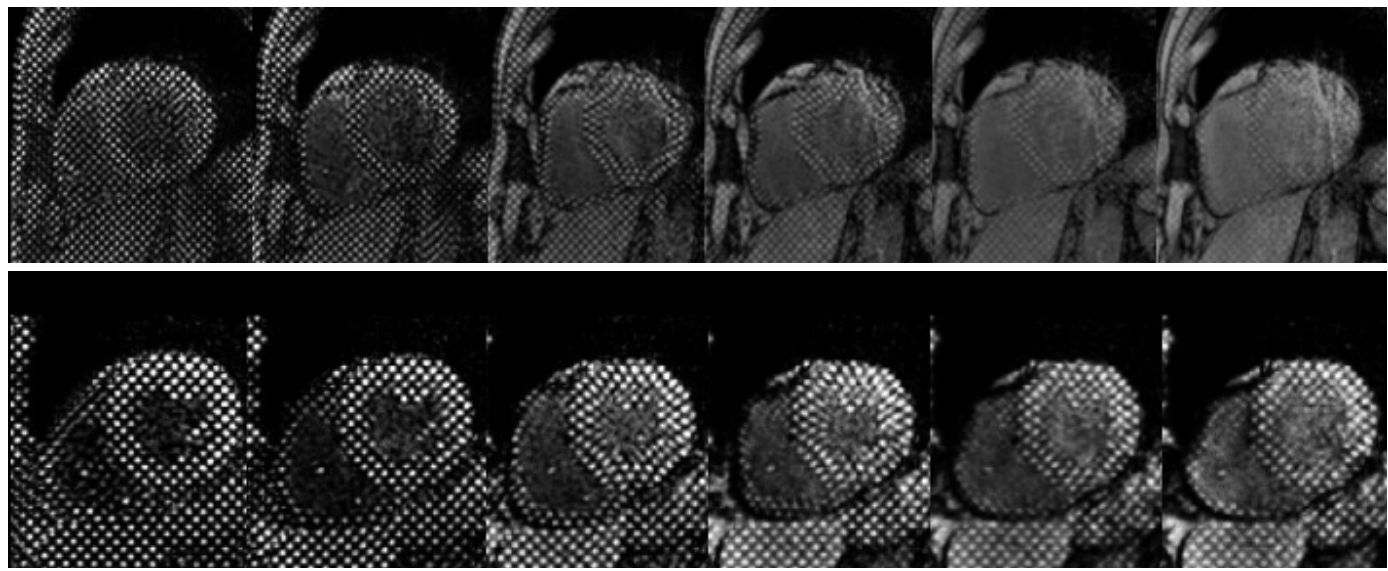

Fig. 1. Short-axis mid-ventricular views of tagged magnetic resonance imaging derived from 1.5 tesla (upper row) and 3.0 tesla (lower row) (left: first frame at end-diastole; center: end-systole; right: last frame at end-diastole). The tagging grid at 1.5 tesla disappears at end-diastole (upper row), whereas the tagging grid at 3.0 tesla persists throughout the cardiac phase on cine imaging (lower row).

\section{TAGGING MRI}

The basic principle behind tagged MRI is to physically tag the myocardium using spatially selective saturation pulses and track its displacement. Tagged MRI has the unique ability to qualify myocardial motion using tag patterns that deform according to the motion of the heart, which aids in tracking motion during contraction and dilation over a series of images [3]. In addition, tagged MRI offers a reproducible measure of regional myocardial strain and can be used for detailed analysis of regional ventricular function with high spatial resolution and accuracy [4]. The accuracy of calculating cardiac strain depends mainly on isolating first-harmonic spectral peaks and correctly evaluating harmonic phase images [5]. Since T1 relaxation time at 3.0 tesla was determined to be longer than that at 1.5 tesla, the tagging grid was maintained at 3.0 tesla throughout the entire cardiac cycle. Visualization of the tagging grid in the lownoise environment of 3.0 tesla was superior to that in 1.5 tesla [6]. An MRI at 3.0 tesla may reduce the phase wrapping effect and correctly isolate the first-harmonic spectral peaks (Figs. 1 and 2).

\section{Assessment of ischemic heart disease}

Combining tagged MRI with late gadolinium enhancement in patients with a prior myocardial infarction revealed contractile impairment and early systolic peaks in peri-infarct areas [7].
These mechanical characteristics can potentially be used to predict the risk of ventricular tachycardia post myocardial infarction. Strain analysis of tagged MRI at 3.0 tesla has enabled in vivo quantification of transmural heterogeneity in myocardial systolic mechanics. Normal contraction is heterogeneous, with subendocardial deformation being markedly greater than subepicardial deformation. In patients with severe coronary artery disease $(\mathrm{CAD})$, resting subendocardial circumferential strain was significantly lower in stenotic coronary segments than in non-stenotic segments (Fig. 3) [8]. The results demonstrated predominant impairment of ischemia-induced subendocardial strain present at rest. Furthermore, MRI tagged for adenosine triphosphate (ATP) stress revealed contractile impairment in the nonischemic, ischemic, and infarcted segments of patients with suspected CAD. Circumferential strain was a factor differentiating ischemic segments from non-ischemic segments under ATP stress, as a greater decrease in circumferential strain was determined for ischemic segments (Fig. 4) [9].

\section{Dyssynchrony in heart failure}

LV dyssynchrony with QRS prolongation, thought to denote intraventricular conduction abnormalities, is present in more than $25 \%$ of patients with heart failure (HF) and has been associated with poor prognosis [10]. Cardiac resynchronization therapy (CRT) has been shown to reduce dyssynchrony and improve symptoms, quality of life, and exercise capacity in HF pa- 


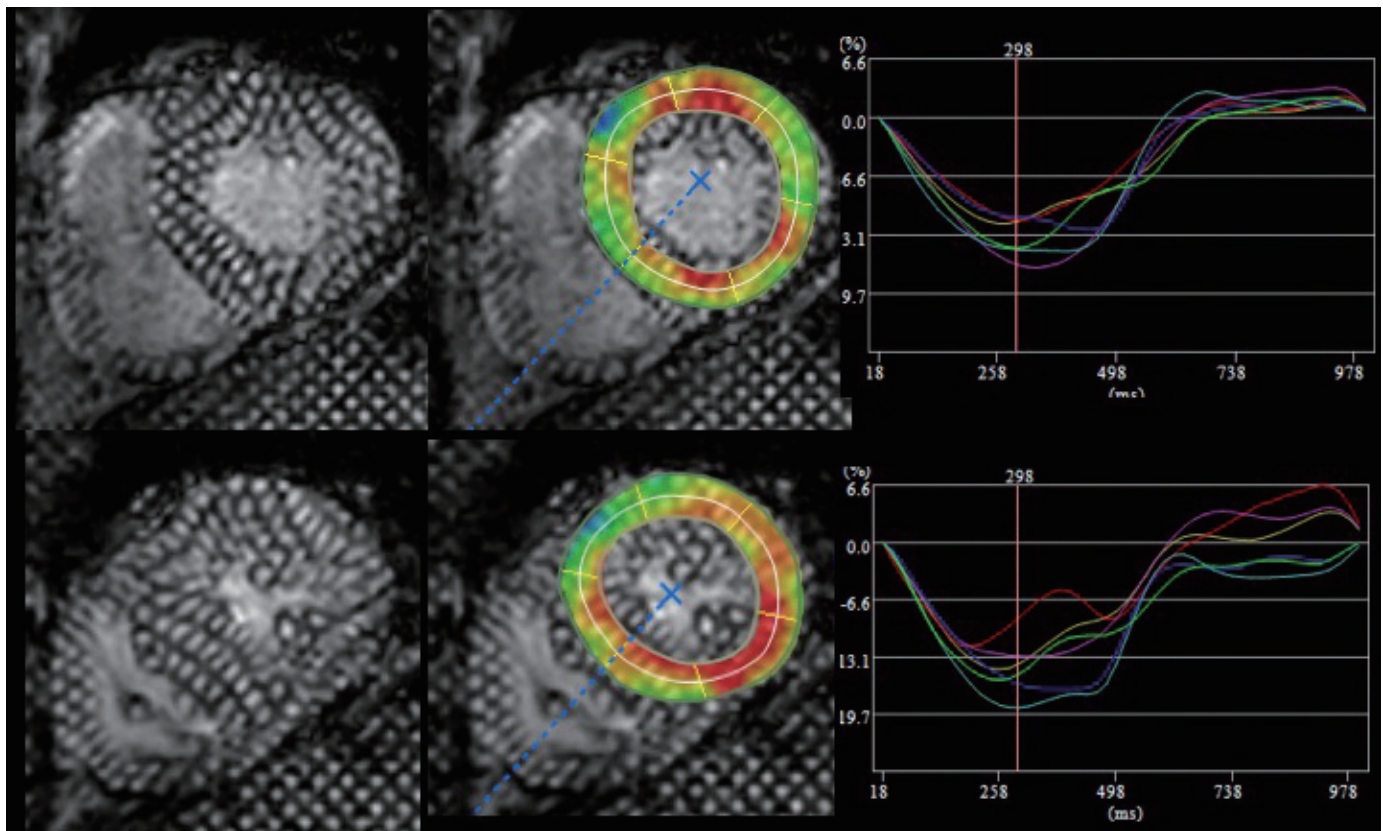

Fig. 2. High temporal tagged magnetic resonance imaging was scanned using a 3.0 tesla magnetic resonance scanner (Achieva $3 T$ Quasar Dual; Philips Healthcare, Best, the Netherlands) with turbo field-echo with a resting grid pulse and a temporal resolution of 50 frames per cycle (left). Strain analysis was performed by a workstation using the two-dimensional pixel tracking algorithm based on feature tracking (Ziostation2, Ziosoft, Inc., Tokyo, Japan) (center). Right graphs are strain-time curves for each myocardial segment.
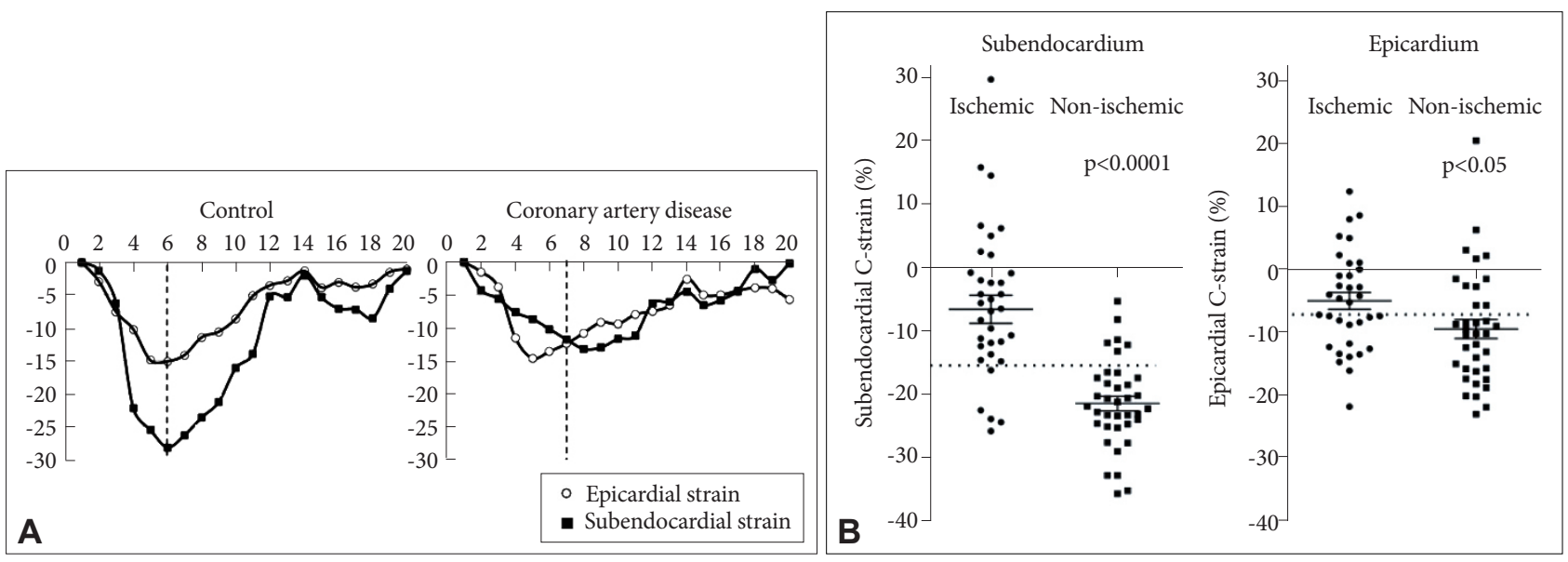

Fig. 3. (A) Healthy control: A man in his 50s (left). Time curves of circumferential strain (C-strain) on an anteroseptal segment as control. In normal contraction, the time curve of subendocardial C-strain shows a great peak at end-systole, while epicardial C-strain is relatively low. A man in his 70s with triple-vessel disease (right). Time curves of C-strain on an ischemic anteroseptal segment. In the ischemic myocardium, the time curves of C-strain show slow slopes and small peaks at end-systole. (B) Scatter plot displaying subendocardial and epicardial $\mathrm{C}$-strain in the stenotic and non-stenotic segments of patients with coronary artery disease. The long horizontal line represents the mean value, and the short upper and lower lines represent the standard error of the mean. The dotted line represents the best cutoff threshold of C-strain. Subendocardial and epicardial C-strain was significantly lower for the stenotic segment than the non-stenotic segment.

tients [11]. Nevertheless, $30-40 \%$ of patients do not respond to CRT [12]. This might be because of imprecise selection criteria, which heavily rely on QRS duration, limitations of current LV dyssynchrony assessment techniques, and lack of guidance for optimal CRT lead positioning [13]. Precise characterization of dyssynchrony is needed to improve the outcome of CRT and help identify the response variations of patients with different HF etiologies to medical therapy. MRI data acquisition is large- ly operator and patient independent and may thus be better suited to characterize dyssynchronous HF and identify appropriate candidates for CRT than Doppler echocardiographic methods (Fig. 5). Tagged MRI is better able to assess spatial dyssynchrony than echocardiographic and radionuclide methods. 123I-Metaiodobenzylguanidine (MIBG) scintigraphy revealed significantly less cardiac sympathetic activity in HF patients with spatial dyssynchrony than in those without, even 

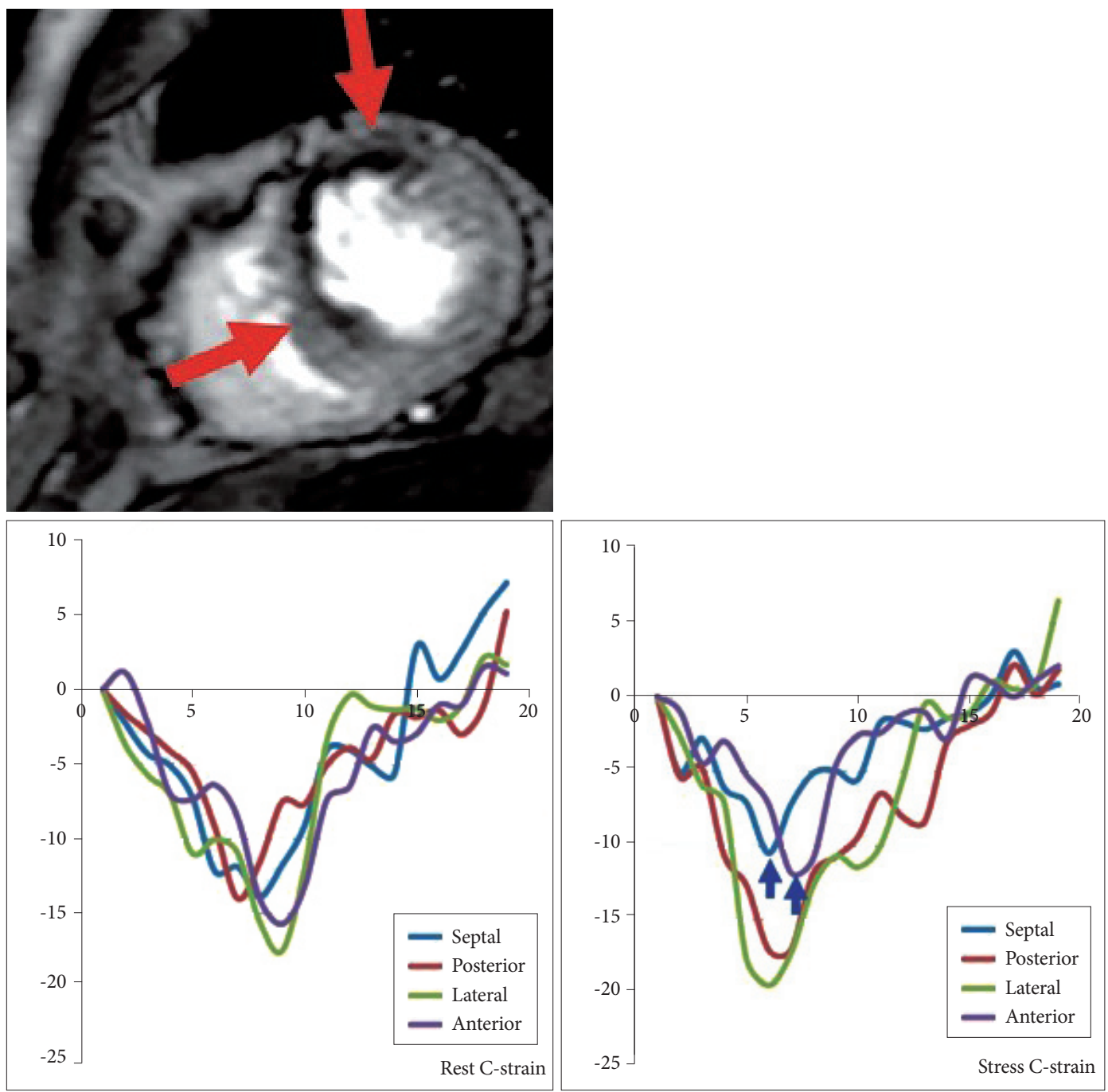

Fig. 4. A man in his 70s with anteroseptal ischemia. An ATP-stress perfusion magnetic resonance imaging detected myocardial perfusion defects in the septal and anterior walls (upper row, red arrows). Perfusion defects were not found in these segments at rest or during late gadolinium enhancement. There was no notable difference in the peak C-strain values between the ischemic (septal and anterior wall) and non-ischemic segments (lateral and posterior wall). However, the absolute peak C-strain value of the ischemic segments decreased under ATP-stress (lower row, blue arrows). ATP: adenosine triphosphate, C-strain: circumferential strain.

though the LVEF of the two groups was not significantly different (Fig. 6) [14]. Quantification of LV dyssynchrony in different geometric planes using cross-correlation analysis of myocardial strain time-curves showed that basal-apical dyssynchrony is an independent predictor of major adverse cardiac events in $\mathrm{HF}$ patients [15].

\section{Applications for adult congenital heart disease and pulmonary hypertension}

Historically, no more than $30-40 \%$ of infants born with congenital heart disease (CHD) have survived until their 10th birthday. However, improved surgical care over the last five decades together with advances in medical management have led to marked declines in the mortality rates of children with $\mathrm{CHD}$ and a new patient population: adults with CHD (ACHD) [16].
ACHD often have right-sided cardiac disease, which has a negative impact on long-term outcomes. Advanced right ventricular (RV) dysfunction and ventricular dyssynchrony are responsible for not only impaired functional capacity, but also lethal ventricular arrhythmias and sudden death [17]. Interventricular dyssynchrony in patients with ACHD was quantified using $\mathrm{RV}$ and LV strain curves derived from short-axis myocardial tagging MRI. Interventricular dyssynchrony correlated significantly with elevations of RV systolic pressure and declines in RVEF and, in addition, offered a more discriminatory parameter to identify RV dysfunction and pressure overload than RV strain [18]. These findings suggest that elevated RV systolic pressure results in significantly delayed RV contraction.

Chronic thromboembolic pulmonary hypertension (CTEPH) is a fatal disease characterized by thrombotic occlusion or nar- 


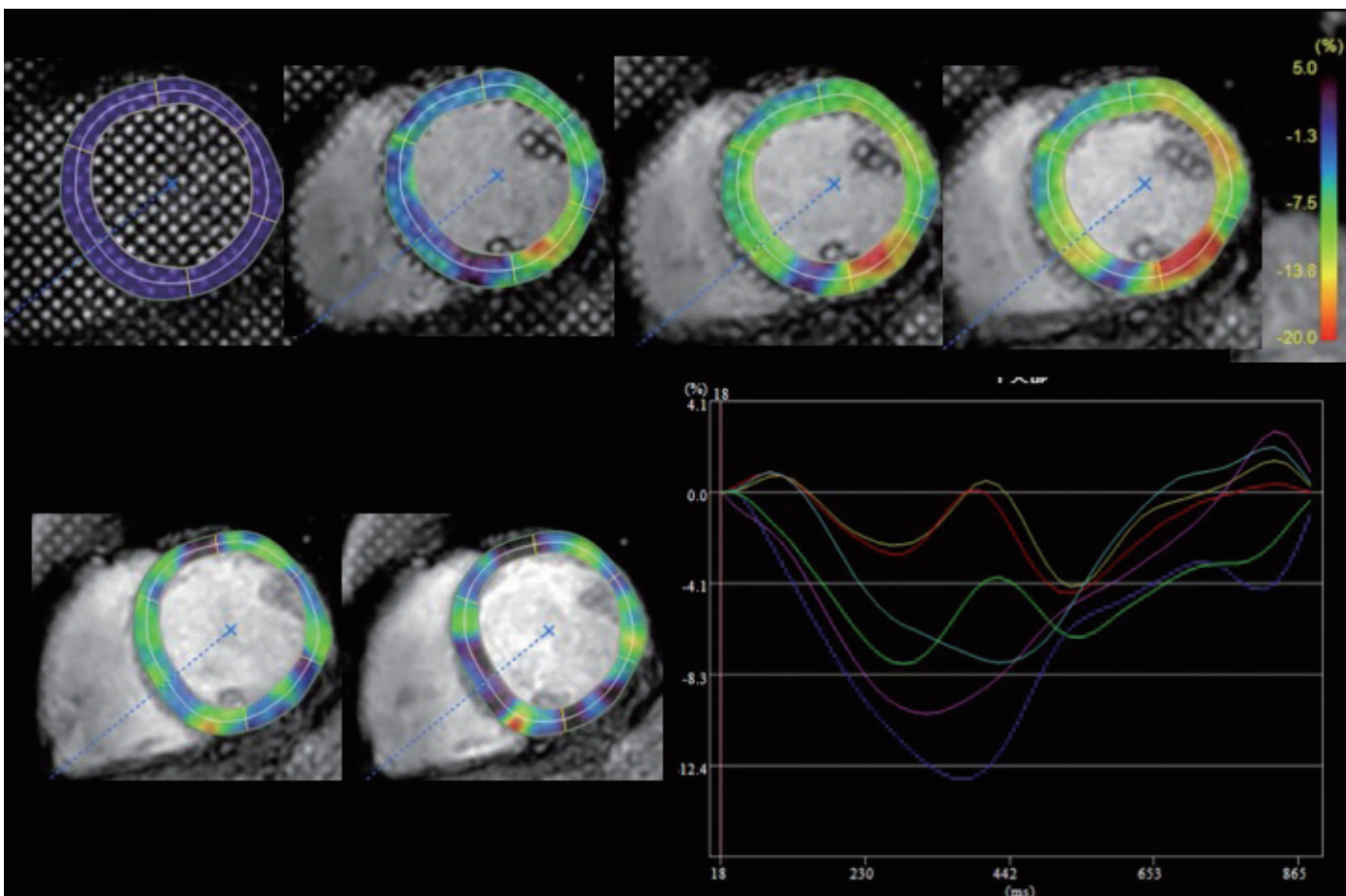

Fig. 5. A man in his 60 s with dilated cardiomyopathy. Color-coded strain map of high temporal tagged magnetic resonance imaging shows heterogeneous strain values in the left ventricle throughout one cardiac cycle (left upper: first frame at end-diastole; right upper: end-systole; lower row: diastole). The time-strain curves of six myocardial segments show variations in the peak time of each strain curve. Left ventricular dyssynchrony is identified (right lower).
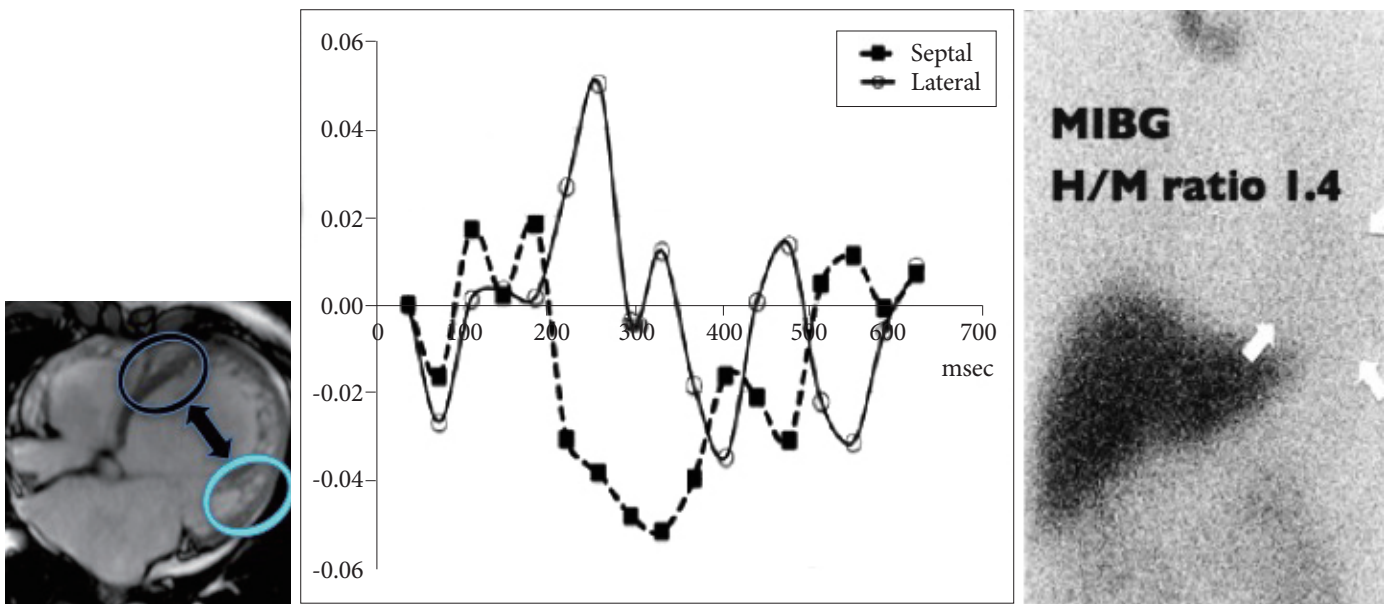

Fig. 6. A man in his 60 s with dilated cardiomyopathy. The strain time curve (center) for the septal segment (left, black circle) has a downward curve and a negative peak during mid- to end-systole, whereas that for the lateral segment (left, blue circle) has an upward curve and a positive peak at early to mid-systole. The systolic peaks for the two strain curves are opposite, indicating spatial dyssynchrony. The anterior view of the thorax on delayed MIBG imaging shows faint accumulation in the left ventricle (arrows). His H/M ratio is decreased to 1.4 (right). H/M: heart-to-mediastinum, MIBG: 123I-Metaiodobenzylguanidine.

rowing of pulmonary arteries, resulting in pulmonary hypertension, right failure, and death [19]. During the past decade, balloon pulmonary angioplasty (BPA) was developed as a therapeutic option for inoperable CTEPH [20]. Yamasaki et al. [21] assessed changes in ventricular strain pattern in patients with inoperable CTEPH before and after BPA using tagged MRI at a high temporal resolution (50 frames/cycle). BPA improved interventricular dyssynchrony, which was strongly associated with increased LV stroke volume. Interventricular dyssynchrony is important for evaluating ventricular interaction, which reduces LV stroke volume and exercise tolerance (Fig. 7).

\section{FEATURE TRACKING OF CINE MRI}

Myocardial tagging acquisition and its requisite post-processing analysis are largely confined to the research environment, 
not least because they are laborious and time consuming. In contrast, FT-CMR is a novel technique that allows quantification of motion and strain using a standard steady-state free-precession sequence, which forms part of a routine LV study protocol.
Whereas myocardial tagging provides a pan-myocardial assessment of deformation, FT-CMR is relatively crude, as it is limited to the assessment of myocardial edges [22]. Tissue tracking technologies such as speckle tracking echocardiography and
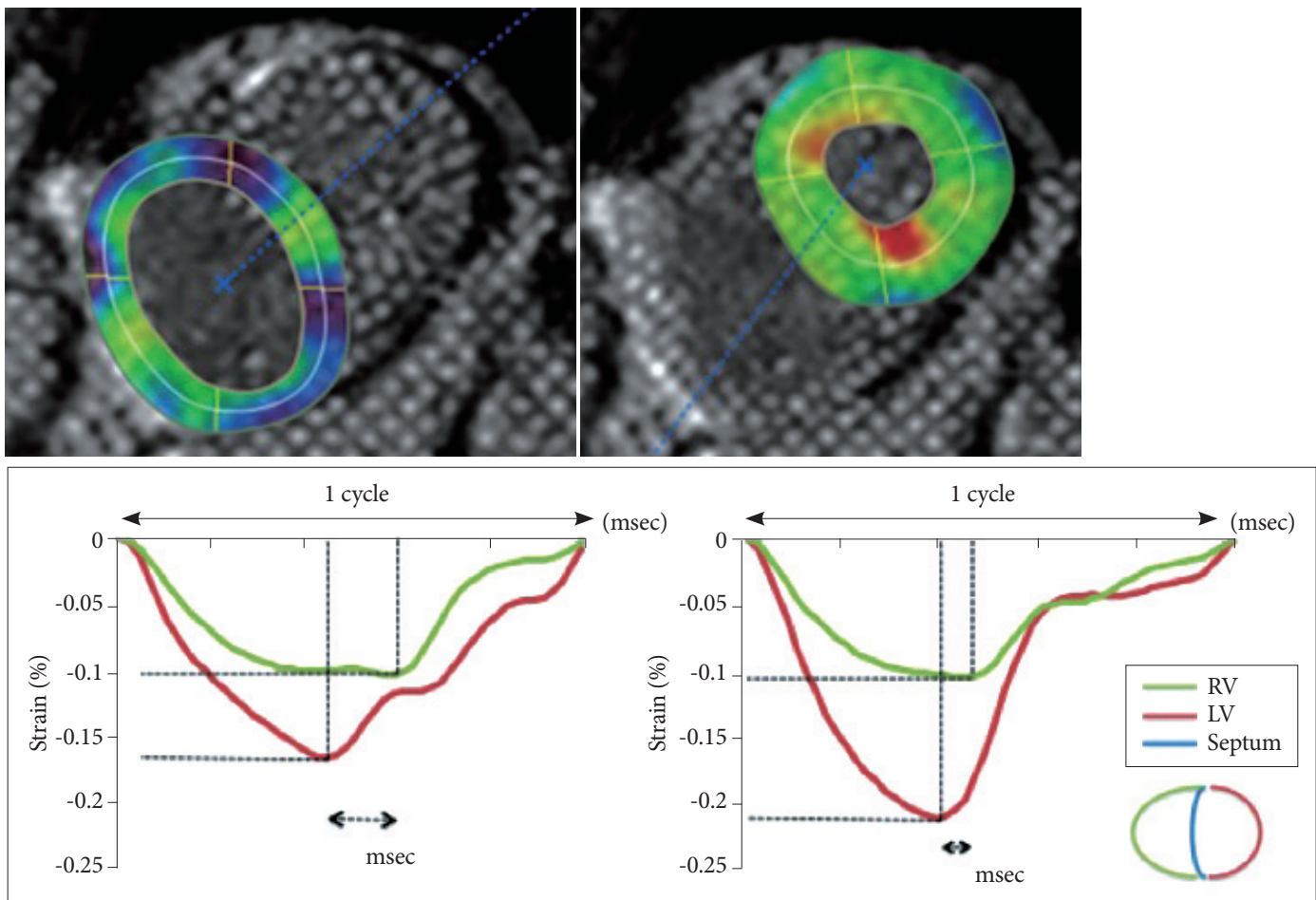

Fig. 7. Color-coded biventricle strain maps for woman in her 60s with chronic thromboembolic pulmonary hypertension (upper row). Strain analysis automatically calculates circumferential strains for the ventricular free walls. Before BPA, the left and right ventricular free walls shorten simultaneously, but the RV reaches its peak later than the LV. After BPA, left and right synchrony has recovered (lower row). BPA: balloon pulmonary angioplasty, RV: right ventricle, LV: left ventricle.

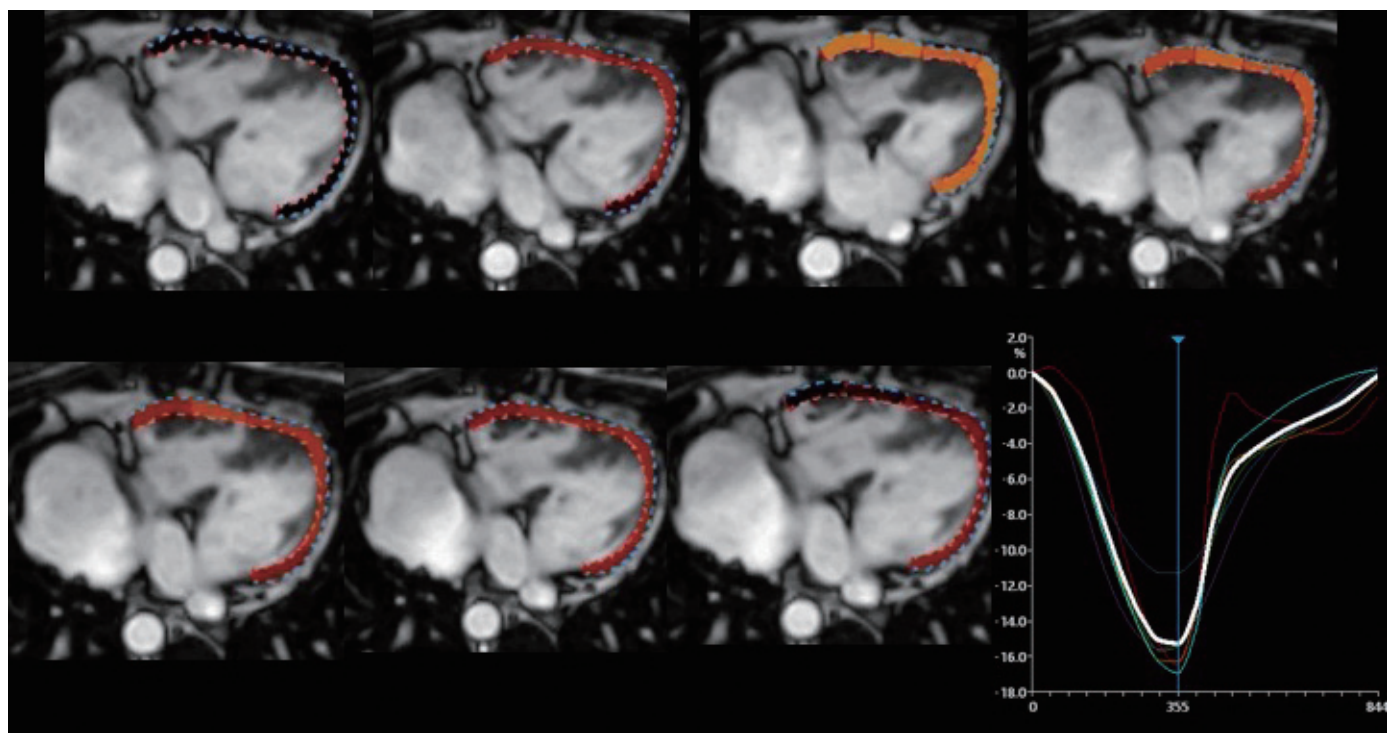

Fig. 8. A woman in her 30s who underwent the Fontan operation to anatomically correct malpositioning of the great arteries, ventricular septum defect, and pulmonary stenosis 30 years prior. Feature-tracking of transaxial cine imaging using available software (Vitrea; Canon Medical Systems Co., Tochigi, Japan) shows color-coded strain values for six myocardial segments throughout one cardiac cycle (left upper: first frame at end-diastole; right upper: end-systole; lower row: diastole). Hot colors represent high strain values; cold colors represent small strain values. The time curves of circumferential strain for six segments and global strain (white) show almost the same peak time. 
FT-CMR rely on identifying a peculiar pattern along a curve on one image, such as the endocardial border, and recognizing the same pattern within a second image taken moments later. This allows for the estimation of myocardial displacement [23]. Kawakubo et al. [24] investigated the clinical significance of evaluating cardiac mechanical dyssynchrony with longitudinal strain analysis using feature tracking of four-chamber cine MRI in HF patients who underwent CRT. LV dyssynchrony could help determine the CRT treatment plan and predict the response of HF patients to CRT [25]. FT-CMR enables comprehensive

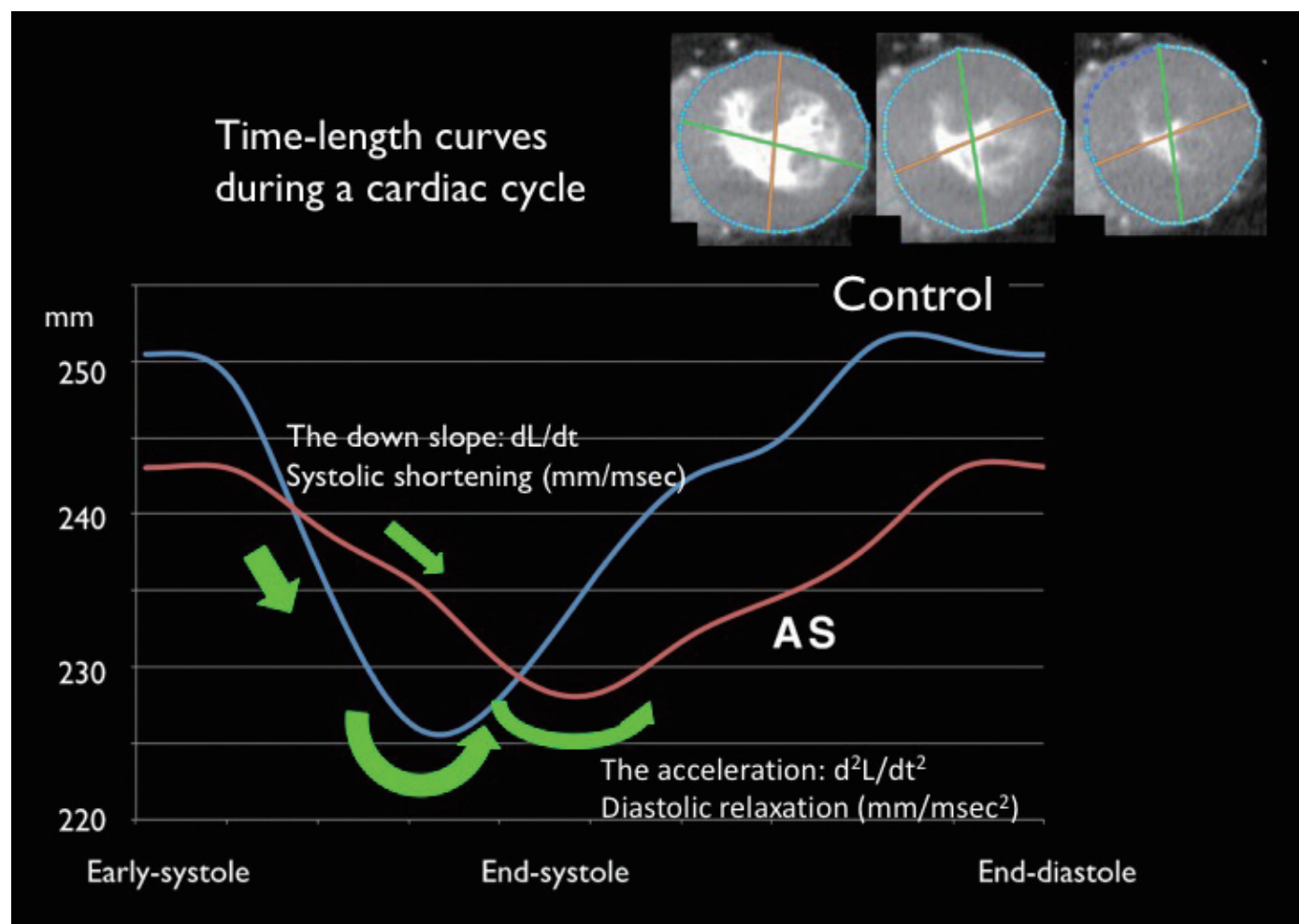

Fig. 9. Calculation of systolic shortening and diastolic relaxation using multi-phase cardiac CT. Motion coherence image processing elicited the epicardial contour on the short-axis cine image of the mid-left ventricle during a cardiac cycle for a woman in her 80s with aortic stenosis (AS) (right upper images). In addition, the circumferential length was measured at 100 phases. Graphs show the time curves of the circumferential length throughout a cardiac cycle for a woman in her 80 s with AS and a man in his 80 s as a control. A shortening ratio of the circumferential length at mid-systole to end-systole is defined as the systolic shortening $(\mathrm{mm} / \mathrm{msec}$, arrows). Acceleration for prolonged circumferential length at end-systole to mid-diastole is defined as the diastolic relaxation $\left(\mathrm{mm} / \mathrm{msec}^{2}\right.$, curved arrows). The diastolic relaxation was obtained by twice differentiating the fitted quadratic equation during end-systole to mid-diastole.
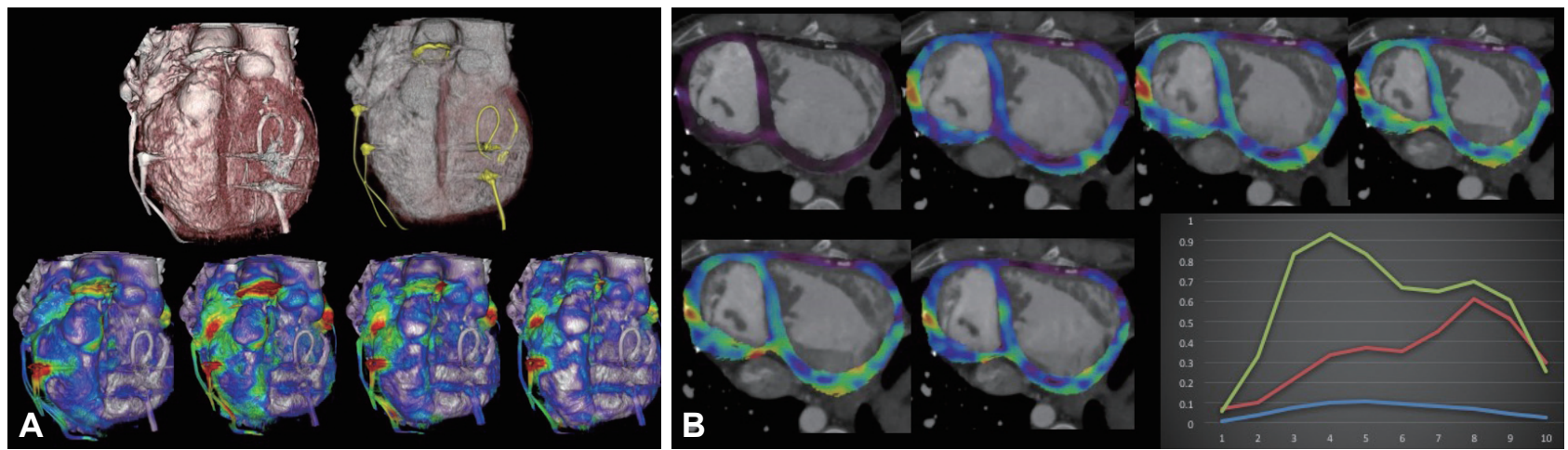

Fig. 10. A woman in her 20s with corrected transposition of great arteries who underwent cardiac resynchronization therapy. (A) Volume rendering images from ECG gate cardiac CT data show implants of pacing leads in the biventricular free wall (upper row). Color maps of 3D maximum principal strain exhibit a hot colored area in the right side of the heart corresponding to the anatomical left ventricle (LV). In contrast, there is no visible strain in the left side, corresponding to the anatomical right ventricle (RV). (B) Transaxial color map of maximum principal strain shows cold colored areas in the anterior wall of the anatomical RV. In contrast, areas of increased strain are visible in the other site of the anatomical RV and the entire anatomical LV (left upper: first frame at end-diastole; right upper: end-systole; lower row: diastole). The time-curves of maximum principal strain show a great strain peak for the anatomical LV at mid-systole (green) and a low peak for the anatomical RV throughout the cardiac cycle. The red line corresponds to the septum. 
calculations of global strain in various types of heart disease. Global longitudinal strain measurements are more reproducible and less variable than other strain parameters and have proven clinical value [26]. FT-CMR-derived global strain measurements can be obtained from patients with various CHDs, including single ventricle physiology and systematic RV in transposition of the great arteries (Fig. 8) [27].

\section{COMPUTED TOMOGRAPHY STRAIN}

Multi-phase cardiac CT with a retrospective ECG gate allows for three-dimensional (3D) volume analysis of global cardiac function with high spatial resolution. Recently, cardiac CT has also shown potential in enabling the quantitative assessment of two-dimensional myocardial strain [28]. CT strain was directly measured from CT volume data without a spatial gap. The motion coherence algorithm was originally introduced as a strategy for noise reduction, which is accomplished by tracking and filtering for unsustainable voxels as image noise [29]. The algorithm can also generate interphase $\mathrm{CT}$ images of two neighboring phases in retrospectively-ECG-gated functional CT data. Quantification of coronary CT angiography demonstrated that aortic stenosis in elderly patients is characterized by verticallongitudinal diastolic dysfunction related to restrictive physiology (Fig. 9) [30]. Tanabe et al. [31] quantified the contractile function of the LV in myocardial infarction using 3D maximum principal strain from multi-phase CT data sets. They reported that peak CT maximum principal strain positively correlated with percentage of systolic wall thickening, as assessed by cine MRI. Patients with metallic devices (e.g., pacemakers and defibrillators) and claustrophobia are considered contraindicated for MRI; therefore, CT strain in a clinical setting is expected for these patients (Fig. 10). On the other hand, CT strain causes the limitations of lower temporal resolution and radiation hazard due to retrospective gate imaging acquisition.

\section{SUMMARY}

Tagging MRI at 3.0 tesla achieves high spatial and temporal resolution, and allows for the detection of subendocardial impairment in ischemia and assessment of inter- and intra-ventricular dyssynchrony. The major advantages of FT-CMR are that it does not require special sequences and can be applied retrospectively. Therefore, it can serve as a valuable and widely used tool to manage patients with various types of heart disease. CT strain derived from multi-phase CT data determines 3D maximum principal strain and is expected to apply for patients contraindicating for MRI due to pacemaker and defibrillator implants.

\section{Conflicts of Interest}

The authors declare that they have no conflict of interest.

\section{Acknowledgments}

This work was supported by the Japan Society for the Promotion of Science (JSPS) KAKENHI (16K10321). We would like to thank Mr. Yuichiro Sano and Mrs. Seiko Shimizu of Canon Medical Systems Co. and Mr. Yamato Shimomiya of Ziosoft Inc. for their technical assistance.

\section{REFERENCES}

1. Buckberg G, Hoffman JI, Mahajan A, Saleh S, Coghlan C. Cardiac mechanics revisited: the relationship of cardiac architecture to ventricular function. Circulation 2008;118:2571-2587.

2. Yeon SB, Reichek N, Tallant BA, Lima JA, Calhoun LP, Clark NR, et al. Validation of in vivo myocardial strain measurement by magnetic resonance tagging with sonomicrometry. J Am Coll Cardiol 2001;38:555-61.

3. Zerhouni EA, Parish DM, Rogers WJ, Yang A, Shapiro EP. Human heart: tagging with MR imaging--a method for noninvasive assessment of myocardial motion. Radiology 1988;169:59-63.

4. Castillo E, Lima JA, Bluemke DA. Regional myocardial function: advances in MR imaging and analysis. Radiographics 2003;23 Spec No:S127S140.

5. Osman NF, Kerwin WS, McVeigh ER, Prince JL. Cardiac motion tracking using CINE harmonic phase (HARP) magnetic resonance imaging. Magn Reson Med 1999;42:1048-1060.

6. Valeti VU, Chun W, Potter DD, Araoz PA, McGee KP, Glockner JF, et al. Myocardial tagging and strain analysis at 3 tesla: comparison with 1.5 tesla imaging. J Magn Reson Imaging 2006;23:477-480.

7. Inoue $Y$, Yang $X$, Nagao M, Higashino H, Hosokawa K, Kido T, et al. Peri-infarct dysfunction in post-myocardial infarction: assessment of 3-T tagged and late enhancement MRI. Eur Radiol 2010;20:1139-1148.

8. Nagao M, Hatakenaka M, Matsuo Y, Kamitani T, Higuchi K, Shikata F, et al. Subendocardial contractile impairment in chronic ischemic myocardium: assessment by strain analysis of 3T tagged CMR. J Cardiovasc Magn Reson 2012;14:14.

9. Kido T, Nagao M, Kido T, Kurata A, Miyagawa M, Ogimoto A, et al. Stress/rest circumferential strain in non-ischemia, ischemia, and infarction--quantification by 3 tesla tagged magnetic resonance imaging. Circ J 2013;77:1235-1241.

10. Kalra PR, Sharma R, Shamim W, Doehner W, Wensel R, Bolger AP, et al. Clinical characteristics and survival of patients with chronic heart failure and prolonged QRS duration. Int J Cardiol 2002;86:225-231.

11. Ståhlberg M, Braunschweig F, Gadler F, Karlsson H, Linde C. Three year outcome of cardiac resynchronization therapy: a single center evaluation. Pacing Clin Electrophysiol 2005;28:1013-1017.

12. Bax JJ, Bleeker GB, Marwick TH, Molhoek SG, Boersma E, Steendijk P, et al. Left ventricular dyssynchrony predicts response and prognosis after cardiac resynchronization therapy. J Am Coll Cardiol 2004;44:18341840.

13. Beshai JF, Grimm RA, Nagueh SF, Baker JH 2nd, Beau SL, Greenberg $\mathrm{SM}$, et al. Cardiac-resynchronization therapy in heart failure with narrow QRS complexes. N Engl J Med 2007;357:2461-2471.

14. Yonezawa M, Nagao M, Abe K, Matsuo Y, Baba S, Kamitani T, et al. Relationship between impaired cardiac sympathetic activity and spatial dyssynchrony in patients with non-ischemic heart failure: assessment by MIBG scintigraphy and tagged MRI. J Nucl Cardiol 2013;20:600-608.

15. Nagao M, Yamasaki Y, Yonezawa M, Kamitani T, Kawanami S, Mukai Y, et al. Geometrical characteristics of left ventricular dyssynchrony in advanced heart failure. Myocardial strain analysis by tagged MRI. Int Heart J 2014;55:512-518.

16. Marelli AJ, Mackie AS, Ionescu-Ittu R, Rahme E, Pilote L. Congenital heart disease in the general population: changing prevalence and age distribution. Circulation 2007;115:163-172. 
17. Warnes CA. Adult congenital heart disease importance of the right ventricle. J Am Coll Cardiol 2009;54:1903-1910.

18. Nagao M, Yamasaki Y, Yonezawa M, Matsuo Y, Kamitani T, Yamamura $\mathrm{K}$, et al. Interventricular dyssynchrony using tagging magnetic resonance imaging predicts right ventricular dysfunction in adult congenital heart disease. Congenit Heart Dis 2015;10:271-280.

19. Lang IM. Chronic thromboembolic pulmonary hypertension: a distinct disease entity. Eur Respir Rev 2015;24:246-252.

20. Lang IM, Madani M. Update on chronic thromboembolic pulmonary hypertension. Circulation 2014;130:508-518.

21. Yamasaki Y, Nagao M, Abe K, Hosokawa K, Kawanami S, Kamitani T, et al. Balloon pulmonary angioplasty improves interventricular dyssynchrony in patients with inoperable chronic thromboembolic pulmonary hypertension: a cardiac MR imaging study. Int J Cardiovasc Imaging 2017;33:229-239.

22. Taylor RJ, Moody WE, Umar F, Edwards NC, Taylor TJ, Stegemann B, et al. Myocardial strain measurement with feature-tracking cardiovascular magnetic resonance: normal values. Eur Heart J Cardiovasc Imaging 2015;16:871-881.

23. Bohs LN, Trahey GE. A novel method for angle independent ultrasonic imaging of blood flow and tissue motion. IEEE Trans Biomed Eng 1991; 38:280-286.

24. Kawakubo M, Nagao M, Kumazawa S, Chishaki AS, Mukai Y, Nakamura Y, et al. Evaluation of cardiac dyssynchrony with longitudinal strain analysis in 4-chamber cine MR imaging. Eur J Radiol 2013;82:2212-2216.

25. Kawakubo M, Nagao M, Kumazawa S, Yamasaki Y, Chishaki AS, Nakamura $\mathrm{Y}$, et al. Evaluation of ventricular dysfunction using semi-automatic longitudinal strain analysis of four-chamber cine MR imaging. Int J Cardiovasc Imaging 2016;32:283-289.

26. Buss SJ, Breuninger K, Lehrke S, Voss A, Galuschky C, Lossnitzer D, et al. Assessment of myocardial deformation with cardiac magnetic resonance strain imaging improves risk stratification in patients with dilated cardiomyopathy. Eur Heart J Cardiovasc Imaging 2015;16:307-315.

27. Shiina Y, Inai K, Takahashi T, Taniguchi K, Watanabe E, Fukushima K, et al. Inter- and intra-ventricular dyssynchrony in the systemic right ventricle is a surrogate marker of major cardiac events in mildly symptomatic patients. Heart Vessels 2018 Feb 28 [Epub]. https://doi.org/10.1007/s00380018-1144-2.

28. Tee MW, Won S, Raman FS, Yi C, Vigneault DM, Davies-Venn C, et al. Regional strain analysis with multidetector CT in a swine cardiomyopathy model: relationship to cardiac MR tagging and myocardial fibrosis. Radiology 2015;277:88-94.

29. Wai B, Thai WE, Brown H, Truong QA. Novel phase-based noise reduction strategy for quantification of left ventricular function and mass assessment by cardiac CT: comparison with cardiac magnetic resonance. Eur J Radiol 2013;82:e337-e341.

30. Nagao M, Yamasaki Y, Kamitani T, Kawanami S, Kondo M, Sonoda H, et al. Geometrical characteristics of aortic root and left ventricular dysfunction in aortic stenosis: quantification of 256-slice coronary CT angiography. Heart Vessels 2017;32:558-565.

31. Tanabe Y, Kido T, Kurata A, Sawada S, Suekuni H, Kido T, et al. Three-dimensional maximum principal strain using cardiac computed tomography for identification of myocardial infarction. Eur Radiol 2017;27:16671675 . 